\title{
Reasons for measles cases not being vaccinated with MMR: investigation into parents' and carers' views following a large measles outbreak
}

\author{
P. MCHALE ${ }^{1 *}$, A. KEENAN ${ }^{2}$ AND S. GHEBREHEWET ${ }^{2}$ \\ ${ }^{1}$ Sefton Council, Bootle, UK \\ ${ }^{2}$ Cheshire \& Merseyside Health Protection Team, Public Health England, Liverpool, UK
}

Received 1 April 2015; Final revision 19 June 2015; Accepted 26 July 2015; first published online 12 August 2015

\section{SUMMARY}

Uptake rates for the combined measles, mumps and rubella (MMR) vaccine have been below the required $95 \%$ in the UK since a retracted and discredited article linking the MMR vaccine with autism and inflammatory bowel disease was released in 1998. This study undertook semistructured telephone interviews among parents or carers of 47 unvaccinated measles cases who were aged between 13 months and 9 years, during a large measles outbreak in Merseyside.

Results showed that concerns over the specific links with autism remain an important cause of refusal to vaccinate, with over half of respondents stating this as a reason. A quarter stated child illness during scheduled vaccination time, while other reasons included general safety concerns and access issues. Over half of respondents felt that more information or a discussion with a health professional would help the decision-making process, while a third stated improved access. There was clear support for vaccination among respondents when asked about current opinions regarding MMR vaccine. The findings support the hypothesis that safety concerns remain a major barrier to MMR vaccination, and also support previous evidence that experience of measles is an important determinant in the decision to vaccinate.

Key words: MMR vaccination, vaccines.

\section{INTRODUCTION}

There are multiple reasons for an individual to be unvaccinated, including individual or parental choice, healthcare access, and contraindication to vaccination [1-4]. However, being unvaccinated leaves an individual susceptible to a host of infections which can result in serious morbidity or mortality. The combined measles, mumps and rubella (MMR) vaccine has particularly high rates of rejection. Introduced in 1988, uptake of MMR vaccine is currently peaking at $92 \%$

\footnotetext{
* Author for correspondence: Dr P. McHale, Sefton Council, 6th Floor, Merton House, Stanley Road, Bootle L20 3DL, UK. (Email: pmchale99@gmail.com)
}

in the UK [5], still below the required levels to confer herd protection.

In 2003, uptake rates were as low as $79 \%$, partly in response to media publicity regarding MMR vaccine following a now discredited and subsequently retracted article in the Lancet in 1998 [6, 7]. This study was widely criticized $[8,9]$ and the Medical Research Council reviewed all the evidence regarding MMR vaccine and its links to inflammatory bowel disease (IBD) in the same year, and concluded that there was no evidence to link MMR vaccine to IBD [10-12]. Furthermore, several studies [13-17] demonstrated that MMR vaccine does not cause autism or IBD. Despite the wealth of literature regarding the safety of MMR vaccine, parental confidence, as 
observed in uptake rates, has been affected, although this has improved in recent years and continues to improve.

In order to understand the factors which may have influenced parents' or carers' decisions not to immunize their children with MMR vaccine, we undertook a descriptive qualitative study using a semi-structured questionnaire that was completed during one-to-one telephone interviews with parents of confirmed measles cases. The aim of the study was to identify factors that continue to affect MMR uptake rates, and consider key issues related to parents or carers of confirmed measles cases.

\section{METHODS}

\section{Participants and setting}

This study was conducted in Merseyside following a measles outbreak in the area [18]. Merseyside has a resident population of about 1.4 million, while first-dose MMR uptake is $>92 \%$ and second-dose uptake is $\sim 85 \%$. This was the largest outbreak in the North West of England since the introduction of MMR vaccine in 1988, with previous outbreaks being sporadic. The outbreak began in January 2012 and was declared closed in September 2013. There were 647 laboratory-confirmed cases and 286 probable cases, and $43 \%$ of confirmed cases were children aged $<5$ years. Twenty-one percent of confirmed cases required hospitalization at time of notification. Cheshire \& Merseyside Health Protection Unit case records were used to identify potential participants and access contact details. Inclusion criteria were: parents or carers of laboratory-confirmed measles cases; cases were aged between 13 months and 9 years (inclusive); cases were eligible for MMR vaccination; and cases had not received MMR vaccination prior to developing measles. This information was validated further during the interview with participants.

\section{Questionnaire and analysis}

A secure online questionnaire was developed in order to collect anonymized data. Attempts were made to contact participants a minimum of three times and a maximum of five times via telephone between 09:00 and 21:00 hours. If participants answered, they were then given information about the caller and reasons for the call using an agreed and standardized script. Verbal consent was obtained from participants and vaccination status was confirmed before progressing with the interview. If participants consented and confirmed that the child was unvaccinated, the interviewer progressed with the interview and completed the questionnaire. All four interviewers received briefing and training with regard to interview process and completion of questionnaires in order to limit interviewer bias.

Questions included covered: receipt of invitation letter; clarity of invitation letter; receipt of reminder letters; whether child has had other vaccinations; whether child has had MMR vaccination since recovery; reasons for not vaccinating with MMR; what would be useful in aiding vaccination; advice to other parents who had not consented to vaccination; and other general comments. Prompts were not given to participants in relation to comments to ensure that their own views were accurately recorded. Responses were analysed using thematic analysis, i.e. responses were analysed and grouped into themes relating to original reasons for not vaccinating.

\section{Ethical standards}

Ethical approval was not required as this study was undertaken as part of the Merseyside measles outbreak investigation. The authors assert that all procedures contributing to this work comply with the ethical standards of the relevant national and institutional committees on human experimentation and with the Helsinki Declaration of 1975 , as revised in 2008.

\section{RESULTS}

\section{Response rate and characteristics of participants}

A total of 227 laboratory-confirmed measles cases were identified as not vaccinated with MMR. Of these, 142 were eligible to be included in the study and contact details were available for 110 cases. Of the 110 cases identified, 30 cases could not be contacted. Of the remaining 80 cases, 23 were excluded as they had already been vaccinated prior to infection, four were too young to be eligible for MMR vaccination at time of infection, two responses were recorded incompletely and four had incorrect identification codes recorded. This left a final sample of 47 cases, 46 of which were from Merseyside and one from Cheshire. Ethnicity was poorly recorded, with $91 \%$ 
Table 1. Responses to questions regarding vaccination of cases and their siblings, including the process

\begin{tabular}{|c|c|c|c|c|}
\hline Question & Yes & No & Unsure & Did not answer \\
\hline Receive a letter? $(n=47)$ & 38 & 4 & 5 & 0 \\
\hline Was the letter clear and informative? $(n=38)$ & 24 & 12 & 0 & 2 \\
\hline Did child have his/her other vaccinations? $(n=47)$ & 40 & 6 & 1 & 0 \\
\hline Did child receive MMR vaccine since recovery? $(n=47)$ & 20 & $25^{*}$ & 2 & 0 \\
\hline
\end{tabular}

* Six children were due to have the vaccine.

(43/47) failing to state their ethnic background. Only 13 cases provided maternal age at birth of child.

In relation to an offer of MMR vaccination, $81 \%$ of participants received a letter of invitation for vaccination, of which $67 \%$ felt it was clear and informative. The majority $(85 \%)$ of the children were up to date with other vaccines. Nearly half $(43 \%, 20 / 47)$ of those questioned had been given the MMR vaccine since recovery and a further six children were due to have the vaccine after the interview (Table 1).

\section{What was the reason for not vaccinating your child with MMR?}

Four general themes arose from the responses: concerns regarding vaccine safety, child illness at time of vaccination, healthcare factors, and other individual factors (Table 2). Twenty-eight responses mentioned safety concerns, the most common reason provided, and of these 23 specifically referred to autism. These responses were mainly just discussing general links to autism; however, eight responses specifically mentioned the role of the media or the Wakefield study [6], while three responses discussed personal experience of autism with friends or family. Four responses discussed concerns about vaccination side-effects or reactions not specified to autism, while one respondent discussed concerns about a mental health risk.

Child illness was the next major factor which influenced the decision of 11 participants. Of these, six provided a firm diagnosis of an illness at the time of vaccination, with the remaining five providing nonspecific illness as a reason for not vaccinating. Specific diagnoses provided included recurrent croup, ear infection, glandular fever, chest infection and 'strep throat'. Four responses discussed healthcare factors, including two who were unable to arrange an appointment for vaccination, one who claimed the vaccine was not offered and one who explained a situation of miscommunication between the local hospital and the General Practitioner (GP). Five response indicated individual factors as the reason for not vaccinating, including forgetting (2), moving house (2) and being unaware of the MMR vaccine on the immunization schedule (1).

Other non-specific reasons included belief in alternative methods such as homeopathy (1), concerns regarding autoimmunity (1), contraindication (1) and concerns over vaccine components (1). Four respondents were unwilling to elaborate on reasons for not vaccinating.

What things would you have found useful to persuade you to vaccinate your child?

When asked 'What things would you have found useful to persuade you to vaccinate your child?' the participants were offered four set responses and 'other'. Twenty-five of 47 agreed 'more information or advice' would improve services, 30 agreed with 'discussion with a health professional', 16 agreed with 'a wider choice of clinics and venues' and 15 agreed with 'earlier or later opening times'. Other responses varied and include the following comments:

- More should be done to make it clearer how serious measles is (3).

- Accessibility to clinics should be easier (1).

- Single vaccines should be available on the NHS (1).

- More information on the benefits of the MMR vaccine (1).

- More information on the invalidity of the Wakefield study (1).

- Take services into the community for those who do not go to their GP (1).

Do you have any advice that you would give now to parents who have not vaccinated their child?

There were 28 responses to this optional question; 22 answered they would recommend vaccination, one was unsure and one would still not recommend MMR vaccination. Other responses included advice 
Table 2. Themes discussed in response to why child did not receive the MMR vaccine

\begin{tabular}{ll}
\hline \hline & No. of \\
Reason given & responses* \\
\hline Safety concerns & $\mathbf{2 8}$ \\
- Autism & 23 \\
- Post-vaccination illness/side-effects/ & 4 \\
$\quad$ reaction & \\
- Mental health & 1 \\
Illness & $\mathbf{1 1}$ \\
- General & 5 \\
- Specific & 6 \\
Healthcare factors & $\mathbf{4}$ \\
- Vaccine not offered & 1 \\
- Unable to arrange appointment & 2 \\
- Miscommunication between services & 1 \\
Other individual factors & $\mathbf{5}$ \\
- Relocation & 2 \\
- Forgot & 2 \\
- Unaware & 1 \\
Other & $\mathbf{8}$ \\
- Unwilling to answer & 4 \\
- Contraindicated & 1 \\
- Belief in homeopathy & 1 \\
- Concerns over autoimmunity & 1 \\
- Unhappy with components of vaccine & 1 \\
\hline
\end{tabular}

* Response count above 47 as some responses discussed multiple themes.

to discuss concerns with a health professional, advice to recognize the seriousness of measles and advice to make the services work for individual needs.

\section{Other general comments}

This was an open question for participants to provide additional information if they wished to do so. Only three participants responded. One respondent was unhappy with the standard of care provided in the local hospital, another felt that health professionals were narrow minded when explaining MMR vaccine and the final respondent commented that parents 'shouldn't be pressured to have vaccinations'.

\section{DISCUSSION}

The main findings in this study support the hypothesis that publicity around the discredited MMR paper remains a major issue influencing parental decision regarding the MMR vaccine. Despite strong evidence and clear information which fully discredited the link to autism, this remains a concern for parents and carers. The high frequency of response to improving services by making more information available and wanting a discussion with a health professional also supports this. Furthermore, two participants had requested for single vaccines to be available through the NHS, indicating the impact of the negative publicity regarding MMR vaccination on parental confidence in the vaccine. The results suggest that other safety concerns are not widely held, with only single responses for mental health risks, post-immunization illness, side-effects and autoimmunity. A large number of respondents indicated that cases were up to date with other childhood vaccines, suggesting the concerns focus solely on the MMR vaccine for many parents and carers. Vaccine safety concerns have previously been demonstrated to have an important influence on parental decision regarding vaccination $[19,20]$, while specific concerns regarding links with autism, immune overload and higher safety of single vaccines have been documented [21, 22].

Although a lack of awareness with regard to the dangers of measles was only stated by one participant as a direct factor in deciding not to take up MMR vaccination, participants were keen to recommend that the dangers of measles should be publicized, and the change in opinion regarding the seriousness of measles following the experience of caring for a child with measles indicate that lack of awareness on the dangers or severity of measles is a significant issue. This is a common thread in vaccination literature, with a low perception of disease severity and risk of contracting the disease associated with lower vaccine uptake $[1,23,24]$. The change seen in vaccination intentions after the outbreak suggests that experience of measles directly influences vaccination beliefs. Studies assessing views on outbreak prevention found conflicting evidence regarding vaccine decliners reconsidering their decision due to an outbreak [1]; however, research has shown an increase in MMR vaccine uptake during a measles outbreak [25].

These findings suggest that safety concerns remain a key area in the decision-making process which is prohibitive to vaccine uptake. Considering the change in beliefs caused by experiences, increasing awareness of measles and its dangers could be beneficial in improving uptake, with media campaigns being potentially useful. Furthermore, the prominence of the historical MMR autism link in decision making would suggest that catch-up campaigns, new methods to disseminate information and opportunistic approaches during outbreaks could be useful [25-27]. Previous evidence supports the use of 
parental meetings to improve the vaccination process [28], with GPs and health visitors viewed as trusted sources of information [29]. The use of personally tailored vaccine invitation letters which are more informative may also be of benefit. However, it is important to recognize that parents who currently reject the MMR vaccine seem to express more complex beliefs which may require novel approaches [30].

Around a third of all participants felt that longer opening hours and a variety of venues would improve services, suggesting access is a concern for parents and carers. However, it is noteworthy that only $9 \%$ of participants highlighted access as a reason for not vaccinating, suggesting that the perception parents have with regard to accessing services does not match with their previous experiences. This could potentially be explained as access issues have been shown to be more prevalent in incomplete vaccination rather than unvaccinated individuals, and the sample does not include incompletely vaccinated children [31]. It is important to recognize that access is an issue which can be directly influenced through policy, and given the economic and health benefits of increased vaccination uptake, it is a clear area of concern. Previous evidence shows that general practice factors have a significant impact on vaccination uptake; practices which had a clear immunization strategy and used a national call/recall system performed significantly better [2].

Almost a quarter of participants stated illness as the reason preventing their children receiving MMR vaccine. Half of these participants responded that the child was unwell for vaccination without a specific diagnosis, which suggests that a clinician or immunizer did not have the opportunity to see the child when they were due for MMR vaccination. This is important as it indicates that parents were making the decision whether or not the child should receive the MMR vaccine and not the healthcare professional. Thus, a number of potentially eligible children may have been denied the MMR vaccine unnecessarily. This is a possible indication of belief that children should not be vaccinated when unwell with even a minor illness [24]. Effective dissemination of information regarding contraindications and reasons to postpone vaccination could resolve this concern.

The sample size achieved is relatively high for a study of this nature, while the setting allows for a unique perspective. To perform the research during and after a large measles outbreak has allowed for a direct investigation of the impact of experience of measles on opinion of vaccination. However, a number of limitations are present. The short and direct nature of the questionnaire allowed for a high number of participants, but did not allow detailed analysis of the reasons provided, or follow-up questions. This would have been particularly valuable in distinguishing causes when illness was provided as a reason for not vaccinating. Additionally, further questions regarding change in views and the impact of direct experience would have been useful. This study was conducted with parents and carers of children who had recently had measles, therefore there is a potential for overstatement bias, as respondents could feel that a somewhat acceptable reason should be provided for their decision not to vaccinate, while the retrospective nature introduces the risk of recall bias.

\section{CONCLUSIONS}

The publicity surrounding the discredited MMR paper suggesting a link between MMR vaccine and autism continues to be a major issue influencing parental decision regarding the MMR vaccine. Information which highlights the safety of the MMR vaccine is readily available; however, this information remains elusive or unconvincing to some parents. The change in views regarding vaccination which occur due to direct experience of measles suggests that, although not a reason to refuse vaccination, highlighting the risks of measles would be an effective approach to improve MMR vaccine uptake. Finally, parents or carers of cases with measles infection perceive access to immunization services and the information that relates to MMR vaccination to be inadequate. This needs to be considered and reviewed regularly by service providers.

\section{ACKNOWLEDGEMENTS}

We thank Cheshire \& Merseyside Health Protection Team staff for their support with conducting the project including maintaining up-to-date data of measles cases.

\section{DECLARATION OF INTEREST}

None.

\section{REFERENCES}

1. Brown KF, et al. Factors underlying parental decisions about combination childhood vaccinations including 
MMR: a systematic review. Vaccine 2010; 28: 42354248 .

2. Lamden KH, Gemmell I. General practice factors and MMR vaccine uptake: structure, process and demography. Journal of Public Health 2008; 30: 251-257.

3. Public Health England. Contraindications and special considerations: the Green Book, chapter 6, 2013 (https://www.gov.uk/government/publications/ contraindications-and-special-considerations-the-green-book-chapter-6). Accessed 17 April 2014.

4. Pearce A, et al. Factors associated with uptake of measles, mumps, and rubella vaccine (MMR) and use of single antigen vaccines in a contemporary UK cohort: prospective cohort study. British Medical Journal 2008; 336: 754-757.

5. Public Health England. Highest MMR vaccine coverage in 25 years, 2013 (https://www.gov.uk/government/news/ highest-mmr-vaccine-coverage-in-25-years). Accessed 14 June 2014.

6. Wakefield AJ, et al. Retracted: Ileal-lymphoid-nodular hyperplasia, non-specific colitis, and pervasive developmental disorder in children. Lancet 1998; 351: 637-641.

7. Friederichs V, Cameron JC, Robertson C. Impact of adverse publicity on MMR vaccine uptake: a population based analysis of vaccine uptake records for one million children, born 1987-2004. Archives of Disease in Childhood 2006; 91: 465-468.

8. Chen RT, DeStefano F. Vaccine adverse events: causal or coincidental? Lancet 1998; 351: 611-612.

9. Nicoll A, Elliman D, Ross E. MMR vaccination and autism 1998. British Medical Journal 1998; 316: 715716.

10. Bower H. MMR vaccine policy is backed. British Medical Journal 1998; 316: 955.

11. Metcalf J. Is measles infection associated with Crohn's disease? British Medical Journal 1998; 316: 166.

12. Fombonne E. Inflammatory bowel disease and autism. Lancet 1998; 351: 955.

13. Patja A, et al. Serious adverse events after measlesmumps-rubella vaccination during a fourteen-year prospective follow-up. Pediatric Infectious Disease Journal 2000; 19: 1127-1134.

14. Peltola H, et al. No evidence for measles, mumps, and rubella vaccine-associated inflammatory bowel disease or autism in a 14-year prospective study. Lancet 1998; 351: 1327-1328.

15. Kaye JA, Melero-Montes MdM, Jick H. Mumps, measles, and rubella vaccine and the incidence of autism recorded by general practitioners: a time trend analysis. British Medical Journal 2001; 322: 460-463.

16. Taylor B, et al. Autism and measles, mumps, and rubella vaccine: no epidemiological evidence for a causal association. Lancet 1999; 353: 2026-2029.
17. Taylor B, et al. Measles, mumps, and rubella vaccination and bowel problems or developmental regression in children with autism: population study. British Medical Journal 2002; 324: 393-396.

18. Vivancos R, et al. An ongoing large outbreak of measles in Merseyside, England, January to June 2012. Eurosurveillance 2012; 17.

19. Alfredsson R, et al. Why do parents hesitate to vaccinate their children against measles, mumps and rubella? Acta Paediatrica 2004; 93: 1232-1237.

20. Bardenheier B, et al. Are parental vaccine safety concerns associated with receipt of measles-mumps-rubella, diphtheria and tetanus toxoids with acellular pertussis, or hepatitis b vaccines by children? Archives of Pediatrics and Adolescent Medicine 2004; 158: 569-575.

21. Casiday R, et al. A survey of UK parental attitudes to the MMR vaccine and trust in medical authority. Vaccine 2006; 24: 177-184.

22. Gellatly J, McVittie C, Tiliopoulos N. Predicting parents' decisions on MMR immunisation: a mixed method investigation. Family Practice 2005; 22: 658-662.

23. Cassell JA, et al. Is the cultural context of MMR rejection a key to an effective public health discourse? Public Health 2006; 120: 783-794.

24. Mills E, et al. Systematic review of qualitative studies exploring parental beliefs and attitudes toward childhood vaccination identifies common barriers to vaccination. Journal of Clinical Epidemiology 2005; 58: 1081-1088.

25. Le Menach A, et al. Increased measles-mumps-rubella (MMR) vaccine uptake in the context of a targeted immunisation campaign during a measles outbreak in a vaccine-reluctant community in England. Vaccine 2014; 32: 1147-1152.

26. Paranthaman K, Bunce A. Opportunistic MMR vaccination for unimmunized children at the time of routine teenage booster vaccination in secondary schools: implications for policy. Epidemiology and Infection 2012; 140: 1612-1616.

27. Nyhan B, et al. Effective messages in vaccine promotion: a randomized trial. Pediatrics 2014; 133: 835-842.

28. Jackson C, et al. Randomised cluster trial to support informed parental decision-making for the MMR vaccine. BMC Public Health 2011; 11: 475.

29. McMurray R, et al. Managing controversy through consultation: a qualitative study of communication and trust around MMR vaccination decisions. British Journal of General Practice 2004; 54: 520-525.

30. Brown KF, et al. U.K. parents' decision-making about measles-mumps-rubella (MMR) vaccine 10 years after the MMR-autism controversy: a qualitative analysis. Vaccine 2012; 30: 1855-1864.

31. Samad L, et al. Incomplete immunisation uptake in infancy: maternal reasons. Vaccine 2006; 24: 6823-6829. 